\title{
Interseccionalidades em Saúde: Predomínio de Sífilis Gestacional em Mulheres Negras e Pardas no Brasil
}

\author{
Tatiane Ribeiro de Morais ${ }^{1}$; Pedro Walisson Gomes Feitosa ${ }^{2}$; Italo Constâncio de Oliveira ${ }^{3}$; \\ Milena Maria Felipe Girão ${ }^{4}$; Wendell da Silva Sales ${ }^{5}$; Eulina Alves Sousa Brito ${ }^{6}$; \\ Liliana Linhares Ribeiro Brito Coutinho ${ }^{7}$; Sally de França Lacerda Pinheiro ${ }^{8}$ \\ Wládia Gislaynne de Sousa Tavares ${ }^{9}$
}

\begin{abstract}
Resumo: A Sífilis Gestacional é responsável por altos índices de morbimortalidade intrauterina, representando um grave problema de saúde pública no grupo materno-infantil. O objetivo desse estudo foi retratar o cenário epidemiológico de sífilis gestacional no Brasil consoantes as variáveis de raça, escolaridade e idade, segundo dados do Boletim epidemiológico publicado pelo Ministério da saúde do Brasil, referentes ao ano de 2017. Para análise comparativa, foram utilizados também dados de 2009 e 2013 publicados na mesma edição. Neste trabalho observou-se predomínio de mulheres com escolaridade inferior a 8 anos; de maioria parda, com idade de 20 a 29 anos. Os dados do presente estudo corroboram com a tese de que a sífilis, assim como outras DSTs, apresentam supremacia em grupos de riscos específicos, sugerindo a necessidade de implementação de políticas públicas preventivas e assistenciais valorizando a democratização e a equidade no cuidado em saúde.
\end{abstract}

Palavras-chave: Sífilis gestacional; Saúde pública; Epidemiologia.

\section{Intersectionalities in Health: Prevalence of Gestational Syphilis in Black Women and Pardas in Brazil}

\begin{abstract}
Gestational Syphilis is responsible for high rates of intrauterine morbidity and mortality, representing a serious public health problem in the maternal-infant group. The objective of this study was to describe the epidemiological scenario of gestational syphilis in Brazil according to the variables of race, schooling and age, according to epidemiological Bulletin published by the Brazilian Ministry of Health for the year 2017. For comparative analysis, 2009 and 2013 data published in the same edition. In this study we observed a predominance of women with schooling below 8 years; of brown majority, aged 20 to 29 years. The data of the present study corroborate with the thesis that syphilis, like other STDs, presents supremacy in groups of specific risks, suggesting the necessity of the implementation of preventive and assistencial public policies valuing the democratization and the equity in the health care.
\end{abstract}

Keywords: Gestational syphilis; Public health; Epidemiology

\footnotetext{
${ }^{1}$ Acadêmico de Medicina da Universidade Federal do Cariri, UFCA, Brasil. tatianee32@ hotmail.com;

${ }^{2}$ Acadêmico de Medicina da Universidade Federal do Cariri, UFCA, Brasil. gomesfeitosa.walisson @ outlook.com;

${ }^{3}$ Acadêmica de Medicina da Universidade Federal do Cariri, UFCA, Brasil. italo.constancio@outlook.com;

${ }^{4}$ Acadêmica de Medicina da Universidade Federal do Cariri, UFCA, Brasil. milenamfg@ hotmail.com;

${ }^{5}$ Acadêmica de Medicina da Universidade Federal do Cariri, UFCA, Brasil. wendellcrato@gmail.com;

${ }^{6}$ Mestranda em Políticas públicas em saúde pelo Instituto Atena. eulinaalvessousabrito@ hotmail.com;

${ }^{7}$ Mestranda em Políticas públicas em saúde pelo Instituto Atena;

${ }^{8}$ Professora adjunta da Universidade Federal do Cariri, UFCA, Brasil. sallylacerda@ hotmail.com;

9 Professora auxiliar da Universidade Federal do Cariri, UFCA, Brasil. wladiatavares@ hotmail.com.
} 


\section{Introdução}

A sífilis é uma doença infecciosa, crônica e de abrangência mundial. Em sua progressão natural, evolui por estágios alternados entre sintomáticos e assintomáticos (SARACENI, 2005). O período de incubação da sífilis decorre entre 10 a 90 dias e sua transmissão ocorre principalmente pela via sexual e verticalmente durante a gestação, e, também, por contato com as lesões (MAGALHÃES et al, 2011).

O agente etiológico da sífilis, a bactéria Treponema pallidum, foi descoberta somente em 1905, pelo zoologista Fritz Schaudin e pelo dermatologista Paul Erich Hoffmann (Brasil, 2010). A sífilis pode se manifestar em diversas formas clínicas, como também em diversos estágios: primário, secundário, latente e terciário, e a sua maior possibilidade de transmissão são nos dois primeiros estágios (PIRES, et al., 2018)

Uma das apresentações clínicas é a sífilis gestacional responsável por altos índices de morbimortalidade intrauterina, com efeitos negativos para o feto e prejudiciais para a gestação, o que retrata um grave problema de saúde pública no grupo materno-infantil (PIRES et al., 2007). A sífilis gestacional decorre da infecção contraída anteriormente a gestação, ou durante a mesma, apresentando menor risco de transmissão para o feto quanto mais antiga for a doença (COSTA et al, 2010).

A presença da doença na gravidez pode ocasionar diversas consequências como nascimento pré-termo, aborto espontâneo, morte intrauterina e óbito perinatal em até $40 \%$ dos casos, inclusive má formações em múltiplos órgãos (DAMASCENO et al, 2014). Consta que cerca de $15 \%$ do total das gestantes manifestam abortos espontâneos no primeiro e no terceiro trimestres de gestação e, para gestantes com sífilis, estima-se que as perdas sejam ainda maiores (RAM, 2001)

Embora a Organização Mundial de Saúde (OMS) ter lançado a diligência em 2007 de eliminar a transmissão de sífilis, atualmente vem ocorrendo o aumento da infecção na gestação (PADOVANI et al, 2018). Até 2016 foram notificados 37.436 casos de sífilis em gestantes, em comparação ao ano de 2015, observou-se um aumento de 14,7\%, denotando a crescente incidência de sífilis na gravidez (BRASIL, 2017). 
Por conseguinte, este artigo objetiva retratar o cenário epidemiológico da sífilis gestacional, consoante dados do Ministério da Saúde referente ao ano de 2017, analisando os aspectos de raça, escolaridade e faixa etária das mulheres acometidas.

\section{Metodologia}

Trata-se de um estudo documental, com base na consulta aos boletins epidemiológicos divulgados pelo Ministério da Saúde referentes ao período de 2017. Esses documentos são elaborados com dados provenientes do Sistema de Informação de Agravos de Notificação (SINAN). Para análise comparativa, foram utilizados também dados de 2009 e 2013 publicados também na referida edição. A incidência de Sífilis gestacional foi apresentada no referido período segundo escolaridade, faixa etária e raça das mulheres acometidas pela patologia e notificadas no período estudado. Posteriormente, os resultados foram discutidos consoante a literatura disponível.

\section{Resultados}

Figura 1: Percentual de sífilis gestacional por faixa etária das mulheres diagnosticadas no ano de 2017

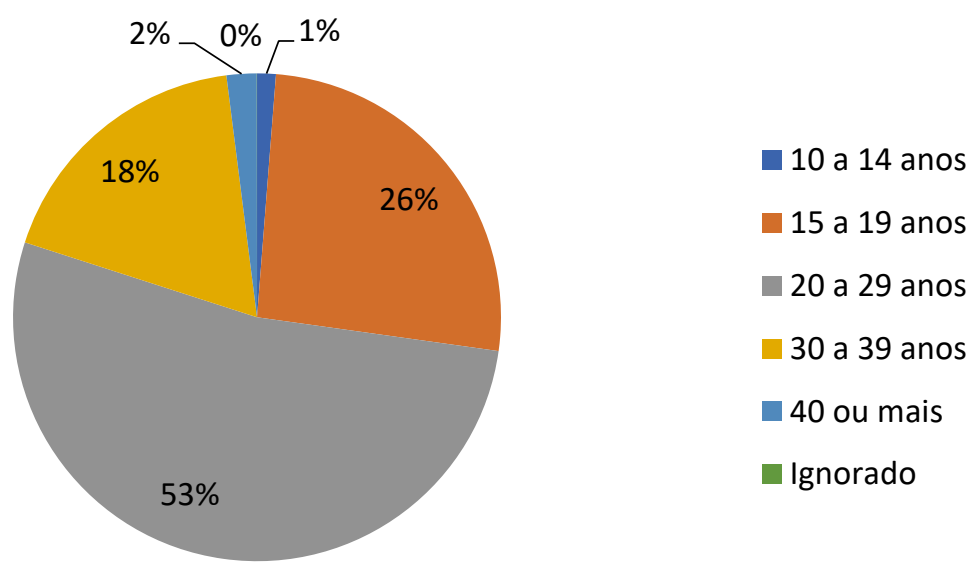

Fonte: SINAN 
A Figura 1 apresenta o percentual de ocorrências de sífilis gestacional por idade das mulheres acometidas, refletindo a supremacia de notificações, 53\%, em mulheres de 20 a 29 anos. Além disso, destaca-se a elevada frequência entre mulheres de 15 a 19 anos, revelando uma relação entre a vida sexual ativa precocemente e o risco de infecções sexualmente transmissíveis.

Figura 2: Gráfico ilustrativo da proporção entre os níveis de escolaridade das gestantes com sífilis ao longo dos anos de 2009, 2013 e 2017.

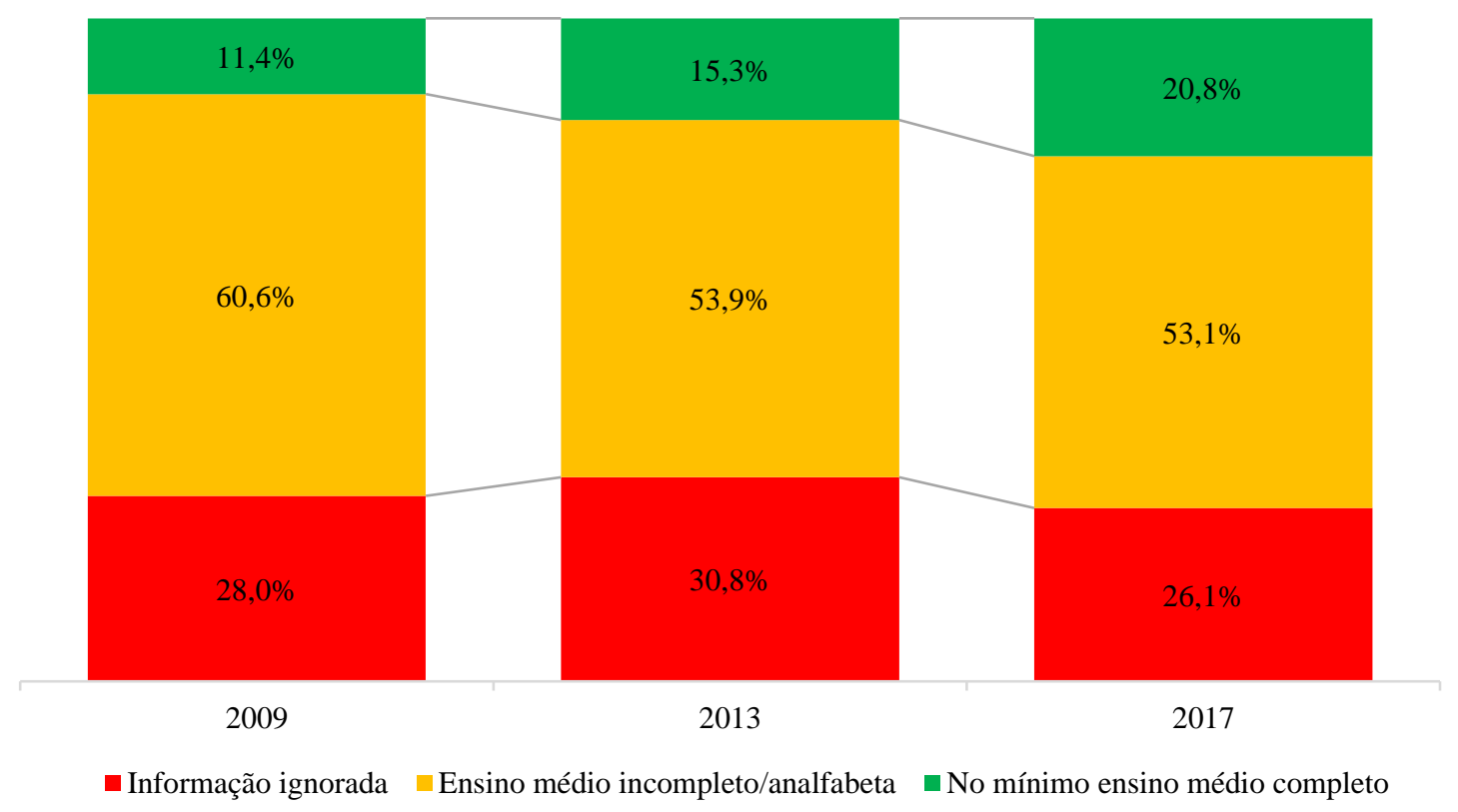

Fonte: SINAN

Na Figura 2 são retratadas as porcentagens referentes aos números de casos de sífilis em gestantes notificados de acordo com o nível de escolaridade desse grupo. Nesse sentido, ao se observar as três faixas analisadas, percebe-se que o grupo das mulheres com ensino médio incompleto ou analfabetas lideram o número de casos nos 3 anos analisados, e, embora demonstre uma leve queda na taxa do total de casos, em números absolutos apresenta crescimento significativo, sendo um aumento de 6.192 casos entre 2009 e 2013 e de 14.760 casos entre 2013 e 2017. 
Entre as grávidas que possuem no mínimo o ensino médio completo, percebeu-se um aumento gradual no decorrer dos anos, consistindo, em 2013, em 2.247 casos a mais do que em 2009, e 6.983 casos em 2017 em relação a 2013. Entre às mulheres que indicaram a opção "informação ignorada", a taxa apresentou pequenas alterações, demonstrando um aumento inicial, saindo de $28 \%$ para $30,8 \%$, e uma posterior queda, partindo de $30,8 \%$ para $26,1 \%$, nos períodos de 2009-2013 e 2013-2017 respectivamente; entretanto, em números absolutos, essa faixa assemelha-se à lógica apresentada pela faixa das mulheres com ensino médio incompleto/analfabetas, tendo um aumento de 4.095 casos no quarto ano posterior à 2009 e de 6.379 entre 2013 e 2017.

Figura 3: Gráfico referente à classificação racial das mulheres diagnosticadas com sífilis na gestação em 2009, 2013 e 2017.

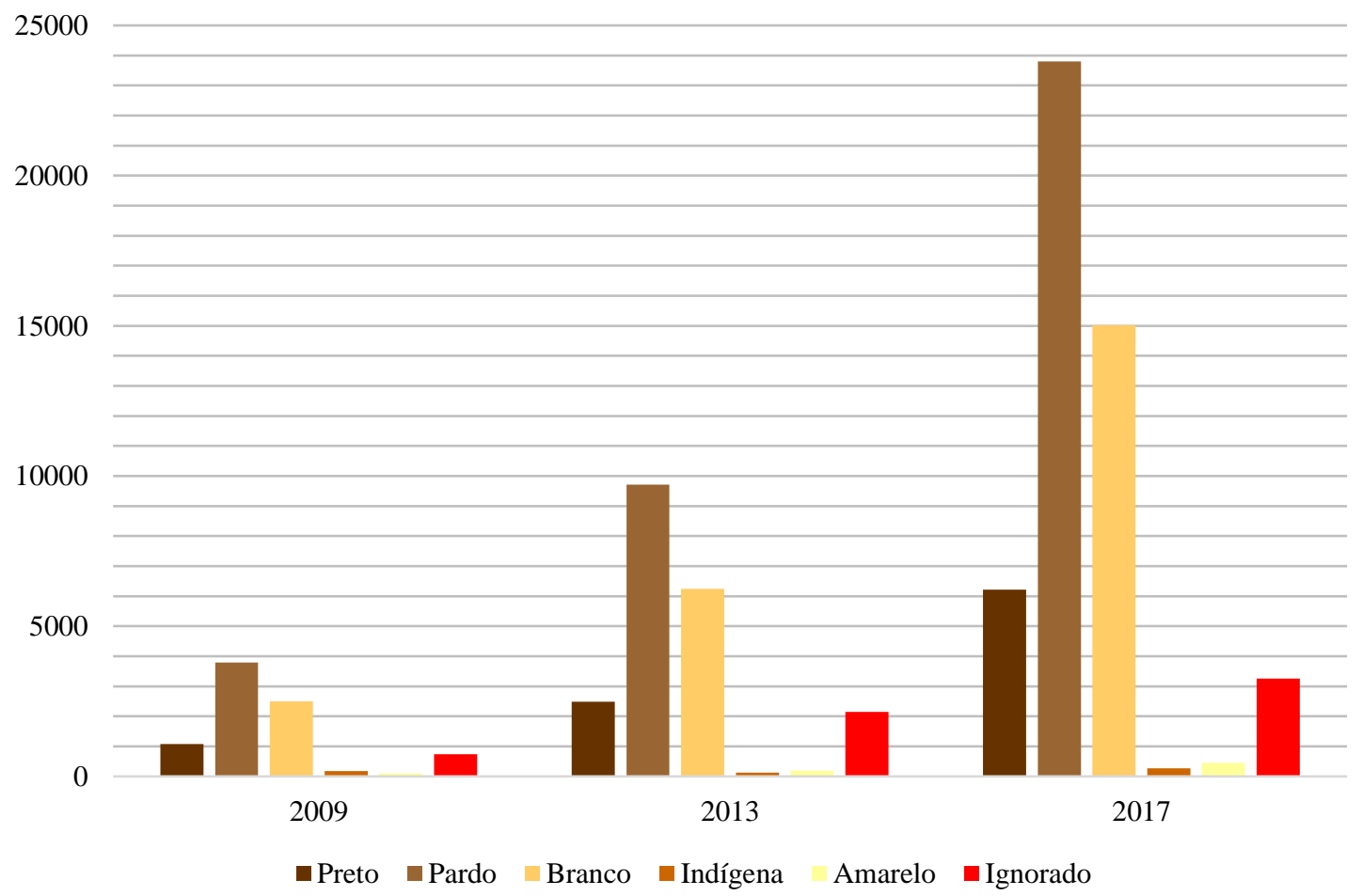

Fonte: SINAN

Com base nos aspectos de raça, a Figura-3 apresenta as taxas de notificações de casos de sífilis em gestantes nos anos de 2009, 2013 e 2017. No ano de 2009 foram notificados 8.367 casos, passando para 20.898 em 2013 e para 49.013 em 2017, o que demonstra um crescimento superior a $100 \%$ entre os períodos supracitados. Desse total, ao somar os 3 anos citados, as 
mulheres pardas se mostraram como as mais afetadas pela doença ao representarem $47,7 \%$ dos casos, seguidas das brancas, com 30,4\%, das pretas, com 12,5\%, das "ignoradas", com 7,84\%, das amarelas e das indígenas, que juntas somam $1,7 \%$ dos casos.

Nesse âmbito, analisando os períodos de 2009-2013 e de 2013-2017 de forma separada, percebe-se que no intervalo que compreende os anos de 2009 a 2013 reflete, grandes taxas de crescimento, sendo o grupo "ignorado" o maior, com 192,4\%, entretanto, excetuando-se a essa lógica, a faixa que abarca as mulheres indígenas obteve uma queda de 25,3\% no número de notificações no mesmo espaço de tempo; já entre o interim referente aos anos de 2013 a 2017, observa-se que todos os grupos em estudo apresentaram aumento no número de casos, tendo a maior taxa de crescimento nas mulheres pretas, com 150,2\%, e o menor nas do grupo “ignorado", com 51,42\%.

\section{Discussão}

A integralidade em saúde, representada como um princípio constitucional, busca apreender as necessidades mais abrangentes dos seres humanos, valorizando a articulação entre atividades preventivas e assistenciais, consoante demandas específicas das populações assistidas. Todavia, o modelo de saúde teórico-conceitual biomédico apresenta-se como um obstáculo à concretização da integralidade. As formações em saúde reverberam como contribuintes ao processo saúde-doença, uma vez que, em suma, não valorizam as populações enquanto seres políticos e epistêmicos, marcados por especificidades relativas aos seus contextos ambientais, econômicos e socioculturais (COELHO et al. 2009; RIGOTTO e AGUIAR, 2017).

Na atenção à saúde das mulheres, a integralidade refere-se como a concretização de uma saúde que garante às mulheres um acesso democrático à saúde por ações resolutivas desenhadas segundo as especificidades do ciclos femininos e do contexto sociocultural e histórico em que as demandas são geradas para cada grupo de mulheres. Seguindo este princípio, a abordagem da população nos sistemas de saúde devem priorizar a escuta sensível de suas necessidades específicas, uma vez que as relações de gênero, raça/cor, classe interferem diretamente na geração de saúde e no processo de adoecimento das mulheres (RIGOTTO e AGUIAR, 2017). 
Todavia, obstáculos para a institucionalização da integralidade do cuidado à saúde da mulher são ainda persistentes no Brasil (COELHO et al. 2009).

Nesse interim, mulheres negras apresentam demandas recorrentes em saúde que o Estado deve analisar ao desenvolver as políticas de saúde para segmentos sociais. Consoante documento do Ministério da Saúde do ano 2005, dados socioeconômicos disponíveis já indicavam que a maioria das mulheres negras encontra-se abaixo da linha da pobreza, representando a seguinte situação: taxa de analfabetismo é o dobro das mulheres brancas; são, em maioria, chefes de família, sem cônjuge e com filhos; por fatores sociais e de discriminação, têm menor acesso aos serviços de saúde de boa qualidade, à atenção ginecológica e à assistência obstétrica - seja no pré-natal, parto ou puerpério; além do maior risco, que as mulheres brancas, de contrair e de morrer mais cedo por determinadas doenças.

A sífilis em gestantes permanece como uma doença a ser prevenida e controlada. Estudos anteriores descreveram características e demandas recorrentes entre as pessoas acometidas: prevalência entre pessoas de raça negra ou parda, idade de 20 a 29 anos, escolaridade inferior a 8 anos, início da atividade sexual precoce e estado civil solteiro (RODRIGUES \& GUIMARÃES, 2004). Outrossim, Guinsburg \& Santos (2010) referem o baixo nível socioeconômico, o uso de drogas e o abandono da escola como fatores associados. Em contra partida, Almeida et al. (2009) apresentaram fatores diversos na caracterização de um perfil crescente de mulheres infectadas por sífilis, sendo estas de maior escolaridade (mais que oito anos de ensino), casadas e relatando apenas um parceiro sexual.

Neste trabalho observou-se, assim como alguns estudos brasileiros, predomínio de mulheres com escolaridade inferior a 8 anos; de maioria parda, com idade de 20 a 29 anos. Os dados do presente estudo corroboram com a tese de que a sífilis, assim como outras DSTs, apresentam supremacia em grupos de riscos específicos, sugerindo a necessidade de implementação de políticas públicas preventivas e assistenciais valorizando a democratização e a equidade no cuidado em saúde.

Destarte, as dificuldades de acesso e qualidade do pré-natal sugerem, portanto, maiores riscos de exposição das crianças de origem social e familiar menos privilegiadas de contraírem Sífilis Congênita. Esta hipótese é fortalecida por dados epidemiológicos do Ministério da Saúde que, além disso, referenciam a evidência de baixo acesso ao pré-natal para as mulheres negras e com baixo nível de instrução (ARAÚJO et al., 2012). 
A ineficácia da assistência posterior à exposição de gestantes, em alguma medida, poderia ser explicada pela dificuldade de uso da penicilina, droga mais custo-efetiva para o tratamento das gestantes com sífilis e dos seus parceiros (GRUMACH et al. 2007). A resistência à efetivação do tratamento da sífilis na rede pública de saúde é evidenciada pela falta de condições técnicas para manejar casos de anafilaxia. Todavia, a mínima incidência de reações letais após o uso da penicilina (1 a 2/100.000) não deve justificar o custo social que a dificuldade de acesso a esse medicamento representa (ARAÚJO et al. 2012).

Dificuldades de prevenção da transmissão vertical persistem, também, pelo obstáculo do diagnóstico e do tratamento precoce adequado. Consoante Araújo et al. (2012), a maioria das gestantes realizou pré-natal, contudo, em $38 \%$ delas o diagnóstico de sífilis foi confirmado apenas no momento do parto ou da curetagem. Segundo Rodrigues e Guimarães(2004), até 40\% dos casos evoluem para essa perda.

Logo, infere-se que a Estratégia Saúde da Família é um instrumento importante de aproximação da população, referido como a ferramenta de entrada para os serviços de saúde, assim como os agentes comunitários de saúde para busca ativa às gestantes ou demais populações infectadas no território. A realização adequada do tratamento da sífilis na gestação é a única maneira de impedir a transmissão vertical e, dessa forma, a infecção congênita (DE LA CALLEN et al. 2013).

\section{Conclusões}

Os dados apresentados referem a necessidade de implantação de políticas e ações em saúde direcionadas às demandas das mulheres em suas diversas nuances, atuando em todos os níveis de complexidade e que atendam às especificidades de populações específicas, como mulheres negras e pardas e de mais vulnerabilidade socioeconômica. Uma vez que, na esfera estatal as políticas de saúde com recorte de gênero e cor são relativamente novas e não suprem aos paradigmas enfrentados por estes grupos de mulheres, refere-se a necessidade imediata do enfretamento destas situações, haja vista o crescimento potencial da casuística de sífilis gestacional, a supremacia de notificações em grupos socialmente menos favorecidos e a ineficácia preventiva e assistencial no sistema público de saúde. 


\section{Referências}

ALMEIDA et. al.. Sífilis em gestantes atendidas em uma unidade de saúde pública de Anápolis, Goiás, Brasil. RBCA 2009; 41(3): 1814.

ARAÚJO, Cinthia Lociks de et al. Incidência da sífilis congênita no Brasil e sua relação com a Estratégia Saúde da Família. Revista Saúde Pública, Brasília, v. 01, n. 01, p.479-486, jan. 2012.

BRASIL. Ministério da Saúde. Boletim epidemiológico sífilis. Secretária de Vigilância em Saúde. Brasil, p. 5-8, 2017.

BRASIL. Ministério da Saúde. Secretaria de Atenção à Saúde. Perspectiva da equidade no Pacto Nacional pela Redução da Mortalidade Materna e Neonatal: atenção à saúde das mulheres negras. Brasília, DF, 2005. Disponível em: <http://bvsms.saude.gov.br/bvs/publicacoes/perspectiva_equidade_pacto_nacional.pdf $>$.

BRASIL. Ministério da Saúde. Súfilis estratégias para diagnóstico no Brasil. Secretaria de Vigilância em Saúde. Brasil, p. 19-20, 2010.

COELHO, Edméia de Almeida Cardoso et al. Integralidade do cuidado à saúde da mulher: limites da prática profissional. Escola Anna Nery, [s.1.], v. 13, n. 1, p.154-160, mar. 2009. GN1 Genesis Network. http://dx.doi.org/10.1590/s1414-81452009000100021.

COSTA, MC et al. Doenças sexualmente transmissíveis na gestação: uma síntese de particularidades. An. bras. dermatol, v. 85, n. 6, p. 776-778, 2010.

DAMASCENO, A. et al. Sífilis na gravidez. Revista Hospital Universitário Pedro Ernesto (HUPE), v. 13, n. 3, 2014.

DE LA CALLEN et al. Syphilis and pregnancy: Study of 94 cases. Med Clin 2013; 141(4): $141-4$.

GUINSBURG e SANTOS. Critérios diagnósticos e tratamento da sífilis congênita. São Paulo: Departamento de Neonatologia, Sociedade Brasileira de Pediatria; 2010. (Documento Científico).

MAGALHÃES, Daniela Mendes dos Santos et al. A sífilis na gestação e sua influência na morbimortalidade materno-infantil: Siphylis in pregnancy and their influence on fetal and maternal morbidity. Com. Ciências Saúde, Brasilia, p.46-46, 2011.

PADOVANI, Camila; OLIVEIRA, Rosana Rosseto de; PELLOSO, Sandra Marisa. Sífilis na gestação: associação das características maternas e perinatais em região do sul do Brasil. Rev. Latino-Am. Enfermagem, Ribeirão Preto, v. 26, e3019, 2018. 
PIRES, ANA CÉLIA SCARI et al. Ocorrência de Sífilis Congênita e os principais fatores relacionados aos índices de transmissão da doença no Brasil da Atualidade - Revisão De Literatura. Revista Uningá Review, [S.1.], v. 19, n. 1, jan. 2018. ISSN 2178-2571. Disponível em: <http://revista.uninga.br/index.php/uningareviews/article/view/1522>. Acesso em: 21 jan. 2019.

PIRES, Osmarina N et al. Vigilância epidemiológica da sífilis na gravidez no centro de saúde do bairro uruará-área verde. DST - J bras Doenças Sex Transm, Santarém, p.162-165, 2007

RIGOTTO, Raquel Maria; AGUIAR, Ada Cristina Pontes. Por que morreu VMS? Sentinelas do des-envolvimento sob o enfoque socioambiental crítico da determinação social da saúde. Saúde em Debate, [s.1.], v. 41, n. 112, p.92-109, mar. 2017. FapUNIFESP (SciELO). http://dx.doi.org/10.1590/0103-1104201711208.

RODRIGUES e GUIMARÃES. Grupo Nacional de Estudo sobre Sífilis Congênita. Positividade para sífilis em puérperas: ainda um desafio para o Brasil. Rev Panam Salud Publica 2004; 16(3): 168-75.

SÁ RAM. Sífilis e gravidez: avaliação da prevalência e fatores de risco nas gestantes atendidas na maternidade escola-UFRJ. DST - J bras Doenças Sex Transm, 13(4): 6-8, 2001.

SANS e GUINSBURG. Prevalência de soropositividade para sífilis e HIV em gestantes de um Hospital Referência Materno Infantil do Estado do Pará. Rev Para Med 2008; 22(3): 1-11.

SARACENI, V. A sífilis, a gravidez e a sífilis congênita. Texto extraído da Tese de doutorado intitulada Avaliação da efetividade das campanhas para eliminação da sífilis congênita, Município do Rio de Janeiro, 1999 e 2000 apresentada ao programa de pós-graduação da Escola Nacional de Saúde Pública da Fundação Oswaldo Cruz, 2005. Modificado pela autora.

\section{Como citar este artigo (Formato ABNT):}

MORAIS, Tatiane Ribeiro de; FEITOSA, Pedro Walisson Gomes; OLIVEIRA, Italo Constâncio de; GIRÃO, Milena Maria Felipe; SALES, Wendell da Silva; BRITO, Eulina Alves Sousa; COUTINHO, Liliana Linhares Ribeiro Brito; PINHEIRO, Sally de França Lacerda; TAVARES, Wládia Gislaynne de Sousa. Interseccionalidades em Saúde: Predomínio de Sífilis Gestacional em Mulheres Negras e Pardas no Brasil. Id on Line Rev.Mult. Psic., 2019, vol.13, n.45, p. 670-679. ISSN: 1981-1179.

Recebido: 11/04/2019

Aceito 06/05/2019 